\title{
Accuracy Validation of Large-scale Block Adjustment without Control of ZY3 Images over China
}

\author{
Yang Bo ${ }^{1}$ \\ Computer School of Wuhan University, Collaborative Innovation Center of Geospatial Technology and \\ State Key Laboratory of Information Engineering in Surveying, Mapping and Remote Sensing, \\ Wuhan University, Wuhan 430072, China \\ 2009106190044@whu.edu.cn
}

Commission I, WG I/4

KEY WORDS: ZY3, Block adjustment, Without control, RPC, Virtual control point, Geometric accuracy

\begin{abstract}
:
Mapping from optical satellite images without ground control is one of the goals of photogrammetry. Using 8802 three linear array stereo images (a total of 26406 images) of ZY3 over China, we propose a large-scale and non-control block adjustment method of optical satellite images based on the RPC model, in which a single image is regarded as an adjustment unit to be organized. To overcome the block distortion caused by unstable adjustment without ground control and the excessive accumulation of errors, we use virtual control points created by the initial RPC model of the images as the weighted observations and add them into the adjustment model to refine the adjustment. We use 8000 uniformly distributed high precision check points to evaluate the geometric accuracy of the DOM (Digital Ortho Model) and DSM (Digital Surface Model) production, for which the standard deviations of plane and elevation are $3.6 \mathrm{~m}$ and $4.2 \mathrm{~m}$ respectively. The geometric accuracy is consistent across the whole block and the mosaic accuracy of neighboring DOM is within a pixel, thus, the seamless mosaic could take place. This method achieves the goal of an accuracy of mapping without ground control better than $5 \mathrm{~m}$ for the whole China from ZY3 satellite images.
\end{abstract}

\section{INTRODUCTION}

Mapping from optical satellite images without ground control is one of the goals of photogrammetry. Currently, with the development of attitude and orbit determination and calibration technology, positioning accuracy from optical satellite images without ground control has reached a higher level of accuracy, which provides a favorable data source for mapping without ground control. Block adjustment utilizes the geometric constraint relationship between homologous image points on adjacent images, thus not only greatly reducing the requirements for ground control points, but also ensuring that all the image products have a consistent geometric accuracy and meet the demand of seamless mosaic of neighboring images, which is a key requirement for large-scale mapping.

In recent years, researchers have carried out a number of block adjustments of optical satellite images based on the development of two adjustment approaches using a single image RPC model (Grodecki and Dial, 2004) or a long strip rigorous model (Rottensteiner et al., 2009; Teo et al., 2010). The former takes a single image as the adjustment unit, compensating for the geometric errors in the image by adding an appropriate mathematical model in the image space

\footnotetext{
${ }^{1}$ Yang Bo (1984- ), male, Ph.D, assistant professor. His research fields are high precision photogrammetric positioning of airborne and spaceborne and geometric processing of satellite remote sensing imagery.
}

RPC model, such as an affine transform (Zhang et al., 2009). The latter is based on the fact that geometric errors in a long image strip are related to the image acquisition time (Zhang et al., 2015; Zhang et al., 2014; Cheng et al., 2010; Srivastava et al., 2008), leading to a corresponding rigorous error compensation polynomial model for attitudes and positions (Poli, 2002; Rottensteiner et al., 2009; Wang, 2001), to compensate for geometric errors in the whole image strip. According to the results of this research, the two methods can achieve almost equal geometric accuracy for data under the same conditions (Tao et al., 2001). Comparing the methods, an adjustment model based on single image RPC has some advantages: the data is more easily organized and since the model is relatively simple, it is more widely used for practical applications. As well, there are advantages of single image RPC model when the scale of block is large.

Tests on data from several popular optical satellites, with sparse ground control points, utilizing the aforementioned two methods and assuming the unknown parameters to be free unknowns in the adjustment, have achieved good results. For instance, SPOT5 (Bouillon et al., 2006) has achieved a positioning accuracy better than $8 \mathrm{~m}$ in plane, IKONOS (Grodecki and Dial, 2003) has reached a 
positioning accuracy better than $1.5 \mathrm{~m}$, and $\mathrm{ZY} 3$ (Wang et al., 2014) has achieved a position accuracy of $3.2 \mathrm{~m}$ and $1.8 \mathrm{~m}$ respectively in plane and elevation with a few control points. Besides, the tests on QuickBird (Passini et al., 2005) and ALOS (Poli, 2002) also have good results. However, when there are no control points, because of the lack of constraints on the unknown parameters, the adjustment model tends to be unstable leading to an ill-conditioned normal equation matrix. This will cause the adjustment to be unstable with an excessive accumulation of errors and distortions in the block (Li, 1982). To solve this problem, the traditional method improves the state of adjustment model by regarding the unknown parameters as weighted measurements according to prior information. However, this method needs to construct error equations and weight for various parameters which contain different physical meanings and are related to each other, so it suffers some limitations in practical applications. In this paper, we propose a large-scale block adjustment method of optical satellite images without ground control based on the RPC model, in which a single image is regarded as an adjustment unit to be organized. This method generates virtual control points using the initial RPC model of each image, and introduce these virtual control points as weighted measurements into adjustment model to improve its stability. Compared with traditional methods, our method uses virtual control points in place of unknown parameters as additional observations, thus avoiding constructing complex adjustment model for unknown parameters and determining their weights. Our method has many advantages: the model is simple and the weights are easy to be determined, so it is effective in practice.

The ZY3 satellite is China's first civilian stereo mapping satellite, equipped with a three linear array panchromatic CCD camera viewing forward, nadir and backward, at an included angle of 22 degrees. Three linear array stereo images are acquired with a swath width larger than $50 \mathrm{~km}$, a nadir resolution of $2.1 \mathrm{~m}$, and forward and backward resolutions of $3.5 \mathrm{~m}$. The base-height ratio for the forward and backward cameras is $0.89(\mathrm{Li}, 2012$; Zhang et al., 2015). After ground geometric calibration and sensor correction, the positioning accuracy of a single image without control is better than $15 \mathrm{~m}(1 \sigma)$, and the inner geometric accuracy is better than 1 pixel (Tang et al., 2012), which is equivalent to the quality of leading international satellites and provides a favorable data source for high precision mapping. By constructing a block of 8802 three linear array stereo images (or a total of 26406 images), covering the whole China which were acquired by the ZY3 satellite from January, 2012 , we have conducted an large-scale and non-control block adjustment test. The results demonstrate the correctness and appropriateness of our method.

\section{METHOD}

\subsection{Block Adjustment Model}

This paper uses the RPC model for the investigation of the ZY-3 satellite data. We represent the RPC model in Eq.(1) as follows.

$$
\left\{\begin{array}{l}
s=F_{x}(\text { Lat }, \text { Lon, Height }) \\
l=F_{y}(\text { Lat }, \text { Lon }, \text { Height })
\end{array}\right.
$$

Where $s$ and $l$ are the image pixel coordinates.

It is necessary to select a suitable mathematical model to describe the distortion of image and attach it into the image space of RPC for the block adjustment, as in the Eq.(2).Geometric distortions can be compensated in each image by additional parameters. Taking ZY3 satellite for example, through the on-orbit geometric calibration and the sensor correction, the remaining geometric distortions in a single image are mainly linear (Wang, 2014; Zhang, 2014; Cao, 2015). Therefore, the image space additional mathematical model chosen is an affine transformation as shown in Eq.(3).

$$
\begin{gathered}
\left\{\begin{array}{l}
l+\Delta l=F_{x}(\text { Lat }, \text { Lon }, \text { Height }) \\
s+\Delta s=F_{y}(\text { Lat }, \text { Lon }, \text { Height })
\end{array}\right. \\
\left\{\begin{array}{l}
\Delta l=a_{0}+a_{1} l+a_{2} s \\
\Delta s=b_{0}+b_{1} s+b_{2} s
\end{array}\right.
\end{gathered}
$$

When establishing the block adjustment model, the original image observations are derived for tie points obtained by automatic matching and the control points obtained by manual semi-automatic measurement. The object space coordinates of the control points are accurately known. Therefore, the undetermined parameters in the error equation include only the image space additional parameters of the RPC model. Obviously, the error equation does not require linearization, and can be rewritten as in Eq. (4):

$$
\left\{\begin{array}{l}
v_{l}=F_{x}(\text { Lat }, \text { Lon, Height })-l-\Delta l \\
v_{s}=F_{y}(\text { Lat, Lon, Height })-s-\Delta s
\end{array}\right.
$$

For the tie points, the undetermined parameters include not only the image space additional parameters of the RPC model, but also the object space coordinates (Lat, Lon, Height), the error equation established for the tie points is nonlinear, and must be linearized assuming appropriate initial values $(\text { Lat, Lon, Height })^{0}$ as in Eq.(5). The initial tie point coordinates can be obtained by intersection using the initial RPC of the corresponding image. 


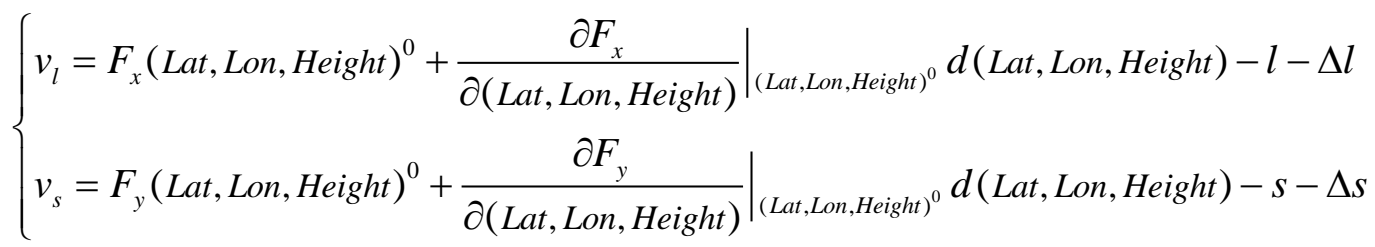

\subsection{Virtual Control Points}

The adjustment parameters can be taken as unconstrained for an adjustment model described above with control. However, when no control exists, the lack of constraints on the control points, will mean that the adjustment model will be relatively uncontrolled, causing an ill-conditioned normal equation matrix. This will lead to instability in the adjustment and excessive accumulation of errors causing distortions in the block. To address this problem, this paper uses the initial RPC model of each image pending adjustment to generate virtual control points. In the following, the method of generation of the virtual control points and construction of observation equations and weights will be described briefly.

Each image to be adjusted is divided into a regular grid. At the center of each grid defined by $p$ (smp,line), using the initial RPC model of the image, on an appropriate elevation datum (for which this paper uses the height offset of initial RPC model), the corresponding object space point $P$ is determined by intersection, In this case, the image point $p$ and the object space point $\mathrm{P}$ constitute a set of virtual control points, as shown.in the Fig. 1.

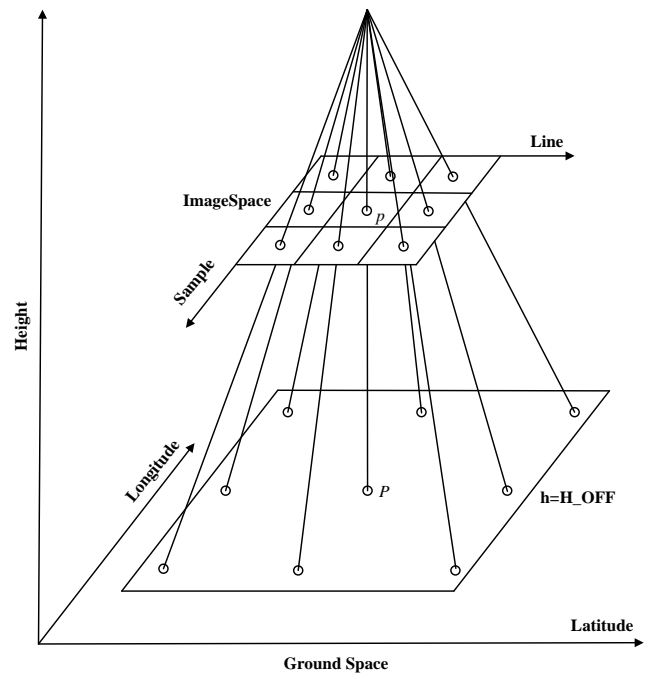

Fig. 1 Generation of virtual control points

Considering the virtual control point as a real control point of a certain accuracy, the error equations can be established directly by Eq.(6) . The observation equations established for all tie points and virtual control points are respectively written in matrix form and shown as Eq.(7) .

$$
\begin{gathered}
V_{v c}=A_{v c} x-L_{v c} \quad P_{v c} \\
V_{t p}=A_{t p} x+B_{t p} t-L_{t p}
\end{gathered}
$$

Let $v c$ and $t p$ represent the virtual control points and tie points; $x$ and $t$ are the adjustment parameters to be solved, which respectively represent the image space parameter vector of the RPC model to be adjusted and object space coordinates correction vector of the tie points. A and B are the partial derivative coefficient matrices in terms of the corresponding unknowns, $L$ and $P$ are the corresponding constant vector and weight coefficient matrix as shown in the Eq.(8). The combined error equation matrices are shown below.

$$
V=A x+B t-L \quad P
$$

Among this : $\quad V=\left[\begin{array}{l}V_{v c} \\ V_{t p}\end{array}\right], \quad A=\left[\begin{array}{c}A_{v c} \\ A_{t p}\end{array}\right]$, $B=\left[\begin{array}{c}0 \\ B_{t p}\end{array}\right], L=\left[\begin{array}{c}L_{v c} \\ L_{t p}\end{array}\right], P=\left[\begin{array}{cc}P_{v c} & 0 \\ 0 & P_{t p}\end{array}\right]$. In the adjustment model, the observations include only two classes: the image points of virtual control points and the image points of tie points. They are independent of each other and can be weighted according to their respective observation accuracy without considering correlations between them. The weights of the image coordinates of the tie point can be set according to the matching accuracy based on SIFT or other high precision matching operators which are usually at sub-pixel level. For the image point observations of the virtual control points, weights can be determined according to the priori information of geometric positioning accuracy of the image without control. Taking the nadir image of the ZY3 satellite for example, long-term on-orbit tests show that the geometric positioning accuracy without control is about $15 \mathrm{~m}$ (RMSE), which amounts in image space according to the geometric resolution, to about 7.5 pixels and thus the weight of the image point observation of the virtual control point is therefore set as $1 /(7.5)^{2}$.

It is worth noting that tie points prove to be "weak" while control points are "strong" in the partial adjustment model, which is caused by the imbalance in the proportions of virtual control points and tie points on the image. This adversely affects the relative geometric accuracy of two adjacent images after adjustment. To avoid this, in this paper we use a scale factor according to the numbers of the two kinds of 
points for each image, i.e., $\mu=N_{t p} / N_{v c}$, where $N_{t p}$ and $N_{v c}$ represent the number of tie points and virtual control points on the image, and the weights $p_{v c}$ of virtual control points in this image are all multiplied by this scale factor.

As can be seen from the above, compared with traditional methods, the proposed method introduces the virtual control points instead of additional observations of the adjustment parameters, which not only improves the rigor of adjustment model, but avoids all kinds of complex adjustment models constructed for unknown adjustment parameters and setting their weights. Our method is simple, easy to set weights with better practicability.

\subsection{Solution of Adjustment}

According to the principle of least squares adjustment, the normal equation is shown as the Eq.(9).

$$
\left[\begin{array}{ll}
A^{T} P A & A_{t p}{ }^{T} P_{t p} B_{t p} \\
B_{t p}{ }^{T} P_{t p} A_{t p} & B_{t p}{ }^{T} P_{t p} B_{t p}
\end{array}\right]\left[\begin{array}{l}
x \\
t
\end{array}\right]=\left[\begin{array}{l}
A^{T} P L \\
B_{t p}{ }^{T} P_{t p} L_{t p}
\end{array}\right]
$$

For a large-scale survey area, the number of the tie points acquired through automatic matching are of the order of tens of thousands in normal circumstances, and the required memory and time is unacceptable if the two unknowns $x$ and $t$ are solved simultaneously. Taking into account that the number of tie points will generally be much larger than the number of images, and more importantly since $B_{t p}{ }^{T} P_{t p} B_{t p}$ is a block diagonal matrix in the normal equation which has excellent properties for matrix storage, computing and other aspects. Accordingly the method commonly used in photogrammetry is to eliminate the object space coordinates of the tie points, and to determine equations for the solution of parameters $x$. On solving $x$, as in Eq.(10), using the initial RPC model parameters of each image and the solved additional model parameters, the object space coordinates of each tie point can be computed by space intersection.

$$
\left[A^{T} P A-A_{t p}{ }^{T} P_{t p} B_{t p}\left(B_{t p}{ }^{T} P_{t p} B_{t p}\right)^{-} B_{t p}{ }^{T} P_{t p} A_{t p}\right] x=A^{T} P L-A_{t p}{ }^{T} P_{t p} B_{t p}\left(B_{t p}{ }^{T} P_{t p} B_{t p}\right)^{-} B_{t p}{ }^{T} P_{t p} L_{t p}
$$

\subsection{Accuracy Evaluation}

It is necessary to evaluate the geometric accuracy of adjustment results. On the one hand, it can be used to indicate whether to increase the control points or tie points in the area with poor accuracy. On the other hand, it can be used to check the quality of the block adjustment, and to find the problems that may exist. The geometric accuracy evaluation of the block adjustment mainly includes two aspects: relative and absolute geometric accuracies. The relative geometric accuracy reflects the consistency of geometry between adjacent images within the block after adjustment, and can be evaluated by making statistics on the mosaic accuracy of adjacent DOMs (Digital Ortho Models). However, a certain number of high-precision check points distributed evenly in the block is needed for the evaluation of the absolute geometric accuracy, which plays an important role in the analysis of error propagation and accumulation, geometric distortion within the block.

\section{EXPERIMENT}

\subsection{Experimental Data}

Based on this method, the paper uses 8802 three linear array stereo images (a total of 26406 images) of ZY3 comprising about 20TB to carry out the block adjustment tests without control. To each image is attached a RPC parameter file. Each image overlaps to a certain degree with adjacent images. The survey area covers about 9 million $\mathrm{km}^{2}$, about $93 \%$ of China's land area except some local areas, such as Guangxi and Guizhou, where valid image data lacks because of unfavorable weather conditions. The covering area contains the plateau, mountains, hills, plains, and desert terrain, the elevation differences are above
8,000 meters. In order to analyze and verify the geometric accuracy of the results of the adjustment, approximately 8,000 high-precision check points (with plan and elevation accuracy higher than $0.1 \mathrm{~m}$ ) were measured by the GPS over the whole country. Fig. 2 (a) and (b) show the spatial distribution of the satellite images and check points.

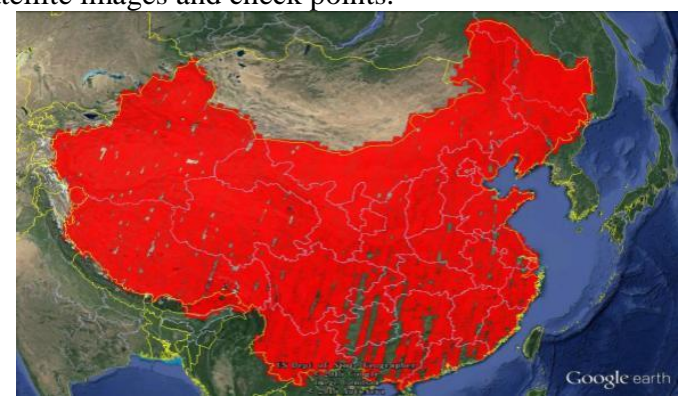

(a) The coverage of Images

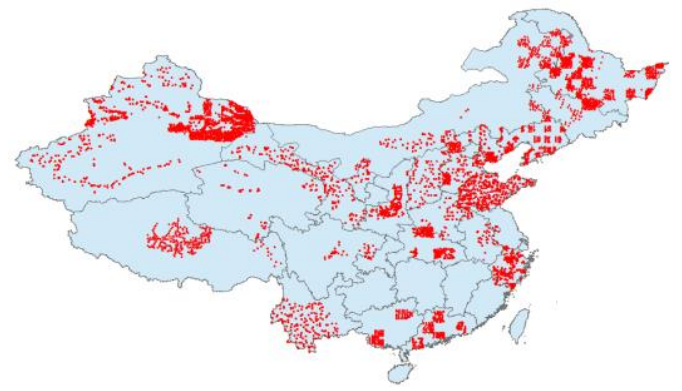

(b) The distribution of ground check points

Fig. 2 The distribution of Experiment data

\subsection{Results and Accuracy Analysis}

We use a cluster computing environment composed of 
100 high performance computing nodes (provided by China Centre for resources satellite data and application, each node has 6-core Xeon-L7455 CPU and $128 \mathrm{G}$ internal memory) for about 2 hours to automatically match the about 3 million uniformly distributed and highly reliable tie points across the whole test area. Each image is divided into $3 * 3$ grids, and virtual control points is generated at the center of each grid based on the method above. Each image has 9 virtual control points, and there is totally 237654 virtual control points in the whole test area. We conducted the block adjustment using the virtual control points and the tie points in a personal computer (Dual-core Intel-15, 8G internal storage) and only two iterations are needed to accomplish the calculation in 15 minutes. The experiment indicates that the adjustment model is refined by introducing the virtual control points, so as to obtain good convergence.

After the adjustment, we use the 8802 three linear array stereo images to create a DSM of $5 \mathrm{~m}$ resolution and DOM of $2 \mathrm{~m}$ resolution. The production data of DOM and DSM is about 20TB. Fig. 3 shows the DSM of the whole test area. Fig. 4 shows the local of DSM of a city and mountains

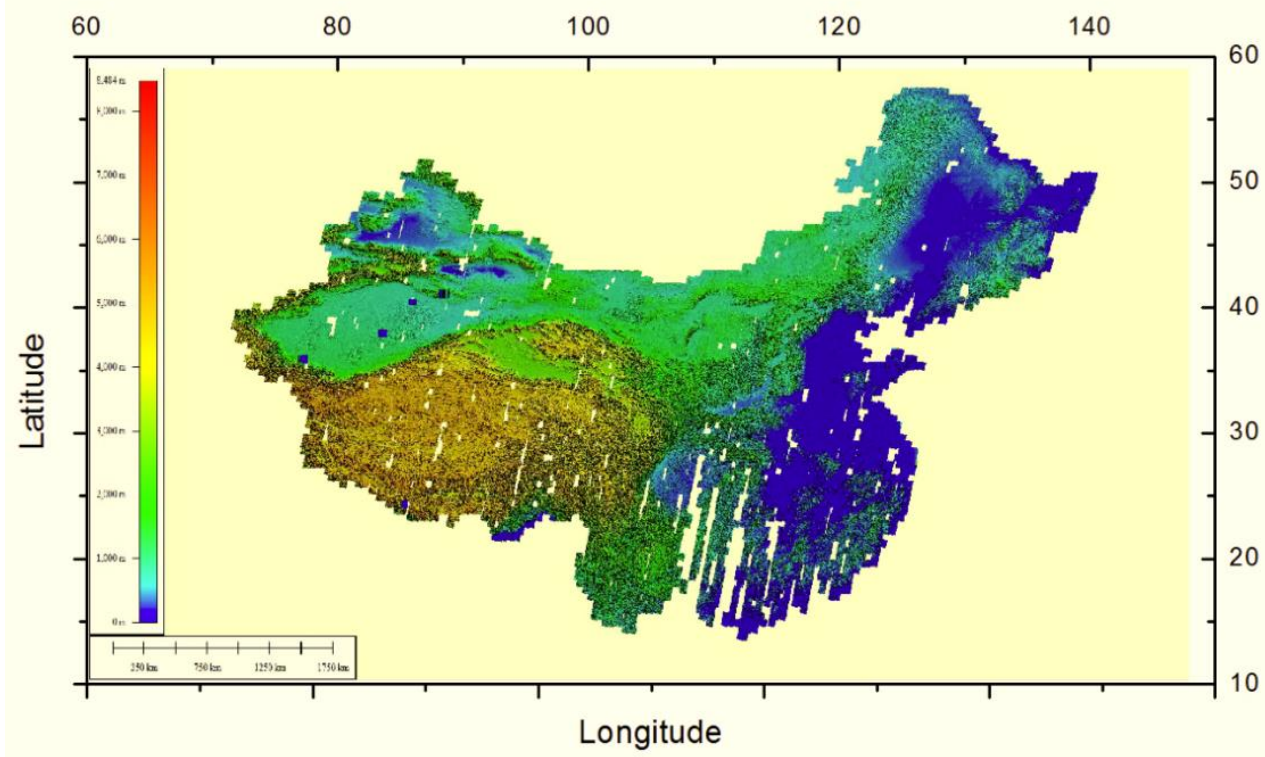

Fig. 3 Nationwide DSM of China

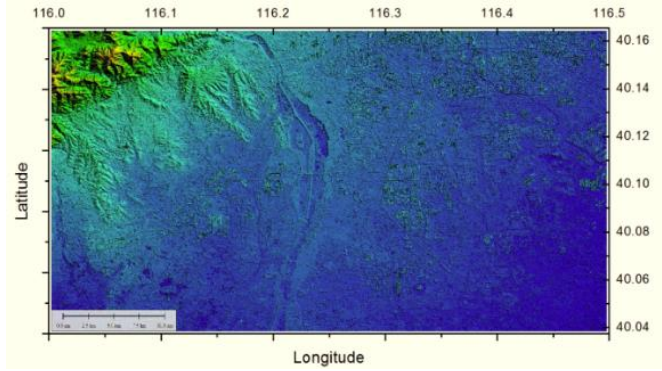

(a) city

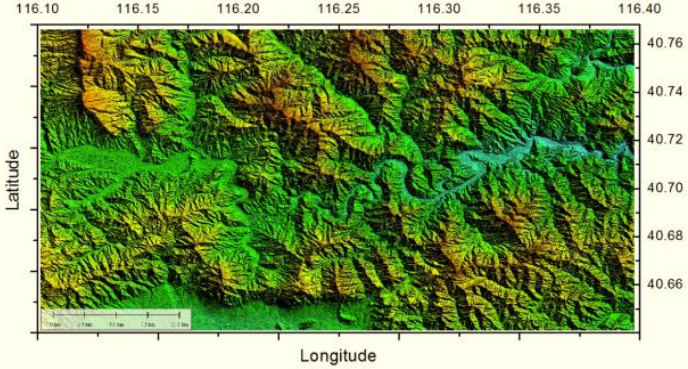

(b) mountain

Fig. 4 DSM of local area

In mapping, the mosaic accuracy between adjacent DOM demonstrates is a key index. 80 pairs of adjacent DOM products are randomly selected for evaluation. We can visually judge the geometric mosaic accuracy of each pair of adjacent DOM of an urban and mountains area from Fig. 5 (a) and (b) respectively which are magnified by 2 times. Moreover, some evenly distributed conjugate points derived by our image matching within the overlapping area of each pair of adjacent DOM. The RMSE of coordinate discrepancy of the conjugate points between two adjacent DOMs are interpreted as the accuracy of geometric mosaic. As shown in Fig. 6, the Y-axis shows the RMSE of geometric coordinate discrepancy of the conjugate points between each pair of DOMs, and X-axis shows the number of pairs of adjacent DOM. 


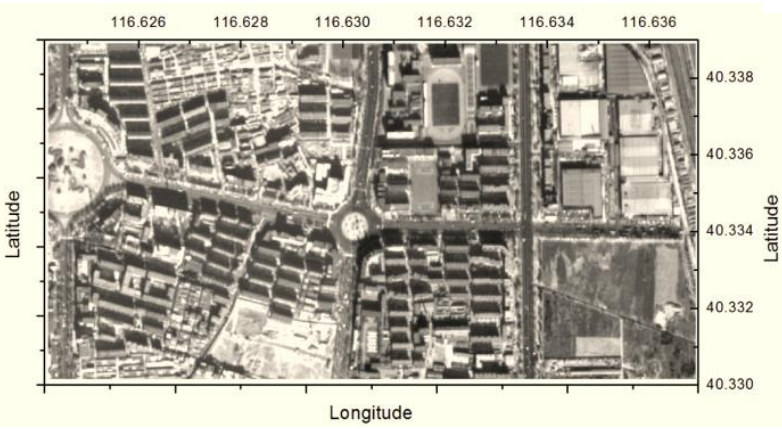

(a) city

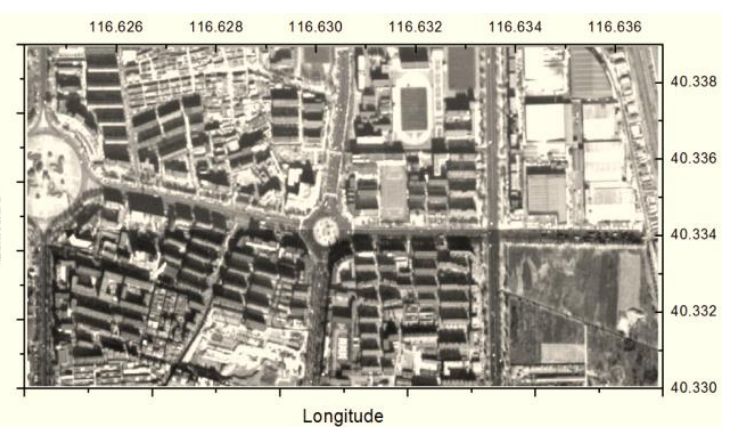

Longitude
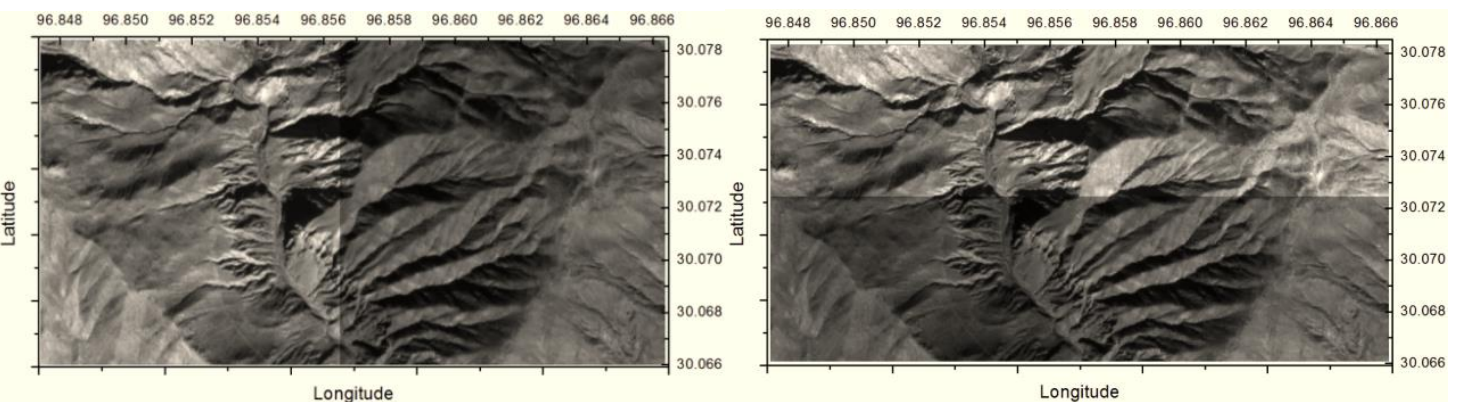

(b) mountain

Fig. 5 Mosaic accuracy between adjacent DOMs

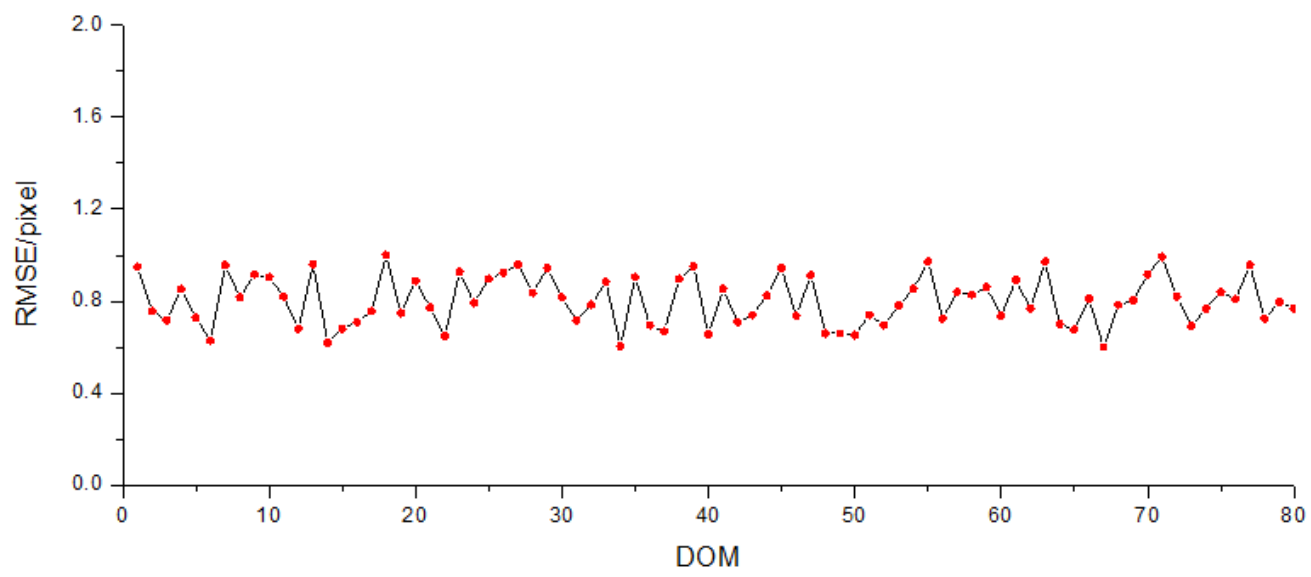

Fig. 6 RMSE of mosaic between adjacent DOMs

From the Fig. 6 and Table 1 we can see that the geometric accuracy of mosaic of adjacent DOM is within 1 pixel, no matter whether it is by visual or quantitative evaluation, so to the accuracy meets the requirements of seamless mosaic.

Apart from the evaluation of the relative geometric accuracy, the above check points can be used to evaluate the plane and elevation geometric accuracy.
In order to analyze the geometric accuracy of the block more scientifically, especially the uniformity of the geometric accuracy, the geometric accuracy of all check points are evaluated. As well, according to the distribution of the checkpoints, the whole area is divided into 5 sub-areas, and the geometric errors of the check points for each sub area is respectively calculated.

\begin{tabular}{ccccccccccccc}
\hline \multirow{2}{*}{ Area } & \multicolumn{4}{c}{ Maximum residual(m) } & \multicolumn{4}{c}{ RMSE(m) } & \multicolumn{3}{c}{ Mean(m) } \\
\cline { 2 - 13 } Global & $\mathrm{X}$ & $\mathrm{Y}$ & $\mathrm{XY}$ & $\mathrm{Z}$ & $\mathrm{X}$ & $\mathrm{Y}$ & $\mathrm{XY}$ & $\mathrm{Z}$ & $\mathrm{X}$ & $\mathrm{Y}$ & $\mathrm{Z}$ \\
West & 4.81 & 5.56 & 9.10 & 9.64 & 2.44 & 2.68 & 3.62 & 4.21 & 0.49 & -0.33 & 0.57 \\
North & 6.12 & 5.86 & 7.08 & 7.67 & 2.76 & 3.12 & 4.16 & 4.41 & 0.62 & 0.71 & 0.23 \\
Middle & 5.65 & 4.98 & 7.53 & 6.03 & 3.08 & 3.12 & 4.38 & 4.67 & -0.64 & -0.57 & -0.87 \\
\hline
\end{tabular}




\begin{tabular}{cccccccccccc}
\hline Northeast & 7.21 & 5.56 & 9.10 & 9.64 & 3.13 & 2.07 & 3.75 & 3.95 & -0.87 & -0.03 & 0.08 \\
East & 4.21 & 3.79 & 5.66 & 6.75 & 2.16 & 2.23 & 3.10 & 3.73 & 0.13 & 0.26 & -0.17 \\
\hline
\end{tabular}

Table 1. Accuracy of geometric error(X: East; Y: North; XY: plane; Z: elevation)

The following conclusions can be drawn:

(1) There are no obvious differences of the mean and RMSE of the residuals in plane and elevation of check points among the five sub-areas.

(2) The mean of the residuals in plane and elevation of check points in each sub-area closes to 0 . There are no obvious systematic offset errors in the block

(3) The plan and elevation maximum errors of each sub-area are less than three times of RMSE

The results show that:

(1) The virtual control points can introduce favorable constraints on the internal error accumulation, which avoids distortion in the block. The accuracy of the center and edges area of block are uniform thus ensuring uniformity of the geometric accuracy within the whole.

(2) Each virtual control point is equivalent to a control point with a certain accuracy. According to adjustment theory, a large number of virtual control points can significantly improve the estimation accuracy of the parameters to achieve an unbiased estimate and provide a simple and practical method for achieving high-level accuracy under the condition where no real control points are used.

(3) The introduction of virtual control points can effectively improve the state of the adjustment model, and avoid problems of unstable and abnormal geometric accuracy of the results, and ensure the adjustment results with high reliability without real control points.

\section{CONCLUSION}

This paper proposes a large-scale and non-control block adjustment method of optical satellite images based on the RPC model, in which a single image is regarded as an adjustment unit to be organized. This method generates virtual control points using the initial RPC model of each image, and introduces these virtual control points as weighted measurements into the adjustment model to realize stable estimation of unknown parameters. Compared with traditional methods, our method introduces virtual control points in place of unknown parameters as attached measurements, so it avoid constructing adjustment model for complex unknown parameters and determining their weights. Our method has many advantages: the model is simple and the weights are easily determined, so it is effective in practice. Based on our method, using 8802 three linear array stereo images (a total of 26406 images), which are obtained by ZY3 satellite and almost covers the whole China, we conduct a block adjustment without ground control and use 8000 evenly distributed high precision check points to evaluate the geometric accuracy of the DOM and DSM production, resulting in a standard deviation of $3.6 \mathrm{~m}$ and $4.2 \mathrm{~m}$ in plane and elevation respectively. The geometric accuracy is even over the whole block and the mosaic accuracy of neighboring DOM is within a pixel. The accuracy thus meets the demand for seamless mosaic. This method achieves the goal that the accuracy of mapping from ZY3 satellite images without ground control is better than $5 \mathrm{~m}$ for the whole China. At present, this method has been successfully used in the commercial satellite image block adjustment software.

\section{ACKNOWLEDGEMENTS}

Professor John Trinder gives many practical advices for the paper and the experiment data and environment are supported by China Center For Resources Satellite Data and Application.

\section{REFERENCES}

Grodecki J, Dial G. Block Adjustment of High-Resolution Satellite Images Described by Rational Polynomials[J]. Photogrammetric Engineering \& Remote Sensing, 2003, 69(1):59-68.

Rottensteiner F, Weser T, Lewis A, et al. A Strip Adjustment Approach for Precise Georeferencing of ALOS Optical Imagery[J]. Geoscience \& Remote Sensing IEEE Transactions on, 2009, 47(12):4083-4091.

R. Passini A, Blades, and K Jacobsen. Handling of large block of high resolution space images[J]. Int.arch.photogramm.remote Sensing, 2005, 36(1):16.

Teo T A, Chen L C, Liu C L, et al. DEM-Aided Block Adjustment for Satellite Images With Weak Convergence Geometry[J]. IEEE Transactions on Geoscience \& Remote Sensing, 2010, 48(4):1907-1918.

Zhang LI, Zhang Jixian, Chen Xiangyang, et al. Block-Adjustment with SPOT-5Imagery and Sparse GCPs Based on RFM[J], Journal of Surveying and Mapping, 2009, 38(4):301-310.

Zhang G, Wang T Y, Li D, et al. Block Adjustment for Satellite Imagery Based on the Strip Constraint[J]. IEEE Transactions on Geoscience \& Remote Sensing, 2015, 53(2):933-941.

Zhang Guo, Wang Taoyang, Li Deren, et al. Block Adjustment for ZY-3 Satellite Standard ImageBased on Strip Constraint[J], Journal of Surverying and Mapping, 2014,43(11):1158-1164.

Cheng Chunquan, Deng Kazhong, Sun Yushan, et all, Study of Block Adjustment for Long-strip Satellite CCD Images[J], Journal of Surverying and Mapping, 2010, 39(2): 162-168.

Srivastava P K, Srinivasan T P, Gupta A, et al. Advanced studies in strip pair processing of Cartosat-1 data[J]. Photogrammetric Record, 2008, 23(123):290-304. 
Poli D. General Model for Airborne and Spaceborne Linear Array Sensors[J]. International Archives of Photogrammetry,2002.

Wang Renxiang, EFP Aerial triangulation for triple linear-array CCD satellite images[J], Science of surverying and mapping, 2002, 27(1):1-7.

Tao C V, Eng P, Hu Y, et al. A comprehensive study of the rational function model for photogrammetric processing $[\mathrm{J}]$. Photogrammetric Engineering \& Remote Sensing, 2001, 67(12):1347-1357.

Bouillon A, Bernard M, Gigord P, et al. SPOT 5 HRS geometric performances: Using block adjustment as a key issue to improve quality of DEM generation[J]. Isprs Journal of Photogrammetry \& Remote Sensing, 2006, 60(3):134-146.

Wang Taoyang, Zhang Guo, Li Deren, et al. Comparison between plane and stereo block adjustment for ZY-3 satellite images[J], 2014, 43(4): 389-395.

$\mathrm{Li}$ Deren. On the signal-to-noise ratio in self-calibrating block adjustment. Journal of Surverying and Mapping 1982.8 11(3) 170-184.

Li Deren, China's first civilian tree-line-array stereo mapping satellite: ZY-3[J], Journal of Surverying and Mapping, 2012, 41(3): 317-322.

Zhang Y, Zheng M, Xiong X, et al. Multistrip Bundle Block Adjustment of ZY-3 Satellite Imagery by Rigorous Sensor Model Without Ground Control Point[J]. Geoscience \& Remote Sensing Letters IEEE, 2015, 12(4):865 - 869 .

Tang Xinming, Zhang Guo, Zhu Xiaoyong, et al. Triple liear-array image geometry model of ZY-3 surverying satellite and its validation[J], Journal of Surverying and Mapping, 2012, 41(2): 191-198.

Wang Mi, Yang Bo, Hu Fen, Zang Xi. On-Orbit Geometric Calibration Model and Its Applications for High-Resolution Optical Satellite Imagery[J]. Remote Sensing. 2014, 6: 4391-4408.

Zhang Y, Zheng M, Xiong J, et al. On-Orbit Geometric Calibration of ZY-3 Three-Line Array Imagery With Multistrip Data Sets[J]. IEEE Transactions on Geoscience \& Remote Sensing, 2014, 52(1):224-234.

Cao J, Yuan X, Gong J. In-Orbit Geometric Calibration and Validation of ZY-3 Three-Line Cameras Based on CCD-Detector Look Angles[J]. Photogrammetric Record, 2015, 30:211-226. 